\title{
A Generic Approach for n-Dimensional Digital Lines
}

\author{
Fabien Feschet and Jean-Pierre Reveillès \\ LAIC Laboratory \\ IUT Clermont-Ferrand - Campus des Cézeaux \\ 63172 Aubière Cedex - France \\ ffeschet, reveil\}@laic.u-clermont1.fr
}

\begin{abstract}
In this paper, we provide an unified view of two definitions of digital lines in 3D via the use of lattice theory and specific projections of the lattice $\mathbb{Z}^{3}$. We use this unified vision to explain the extension of the definition of Voss 1 to an arbitrary dimension and we show how to extend the definition of Figueiredo and Reveillès [2] to an arbitrary dimension.
\end{abstract}

\section{Introduction}

Digital lines are among the simplest primitives in Digital Geometry. Many definitions have been proposed by many authors [3, which are almost all equivalent in 2D. Several drawing algorithms are known as well as several recognition algorithms. All of this explains why digital lines are extremely central for a lot of digital algorithms. Thus, it is natural to look for an extension of the definition of 2D digital lines to 3D digital lines. Moreover, as the applications nowadays manipulate 3D, 4D and sometimes higher dimensional data, extensions of digital lines to n-D becomes also very important and critical.

Several extensions have been proposed to define 3D digital lines. First, Voss [1] recalled some previous works by Kim 4] and proposed a definition of n-D digital lines based on the integer part function $\lfloor$.$\rfloor . Second, the work of Debled-$ Rennesson et al. [56] proposed to define 3D digital lines through their projections (two or three in the general case) on the planes defined by the axes of the standard basis of $\mathbb{Z}^{3}$, and used the arithmetical approach of Reveillès [7. It must be noticed that [4] also used projections onto the coordinates planes. The work of Debled et al [6] also leads to a recognition algorithm. A third approach was also done by Figueiredo and Reveillès in [8, 2, using lattice theory and projections onto the orthogonal plane of a direction $v$ in $\mathbb{Z}^{3}$. As it can be seen, only the definition given by Voss [1] extends to an arbitrary dimension. Beside this, we can note that there exist drawing algorithms of digital lines in n-D [9] based on displacement vectors. Moreover, the definition of $n-D$ digital lines is related to the notion of digitization. Some models are presented by Klette [10] (with the important correction given in [1] ]).

The goal of this paper is to present a unified and generic view of the definition of Voss [1] and the definition of Figueiredo and Reveillès [2]. Moreover, due 
to this unified viewpoint, we also extend the last definition to an arbitrary dimension. The main mathematical tools used in this paper are lattice theory and projections of lattices. We prove in the paper that both definition are obtained via projection of the lattice $\mathbb{Z}^{3}$ onto specific planes which are the $x O y$ plane for the definition of Voss and the orthogonal plane - as it was already the case - in the definition of Figueiredo and Reveillès.

The structure of the paper is as follows. We first recall how to manage symmetries in $3 \mathrm{D}$ via the octaedral group in section 2 . This is followed in section 3 by the construction of the definition of Voss in dimension 3, as well as a recall of the construction of Figueiredo and Reveillès. We end this section by the presentation of a drawing algorithm. Then in section 4, those approaches are extended to an arbitrary dimension. We also present some results concerning the basis of the lattice we manipulate. The paper ends in section 5 with some conclusions and perspectives.

\section{Preliminaries}

In $2 \mathrm{D}$, it is usual to restrict the study of digital lines to the first octant where for each point $(x, y)$, we have $0 \leq y \leq x$. In higher dimension, we can do the same following the approach of Reveillès [12. Hence, we will use the group of the symmetries of the unit cube in $3 D$. We denote this octaedral group by $O_{h}$. This group can be identified to the product of the group $\left(\frac{\mathbb{Z}}{2 \mathbb{Z}}\right)^{3}$ of order 8 , and the group $\mathcal{S}_{3}$ of the permutations of the three letters $a, b, c$, whose order is 6 . The order of $O_{h}$ is thus 48. Its geometrical interpretation is easy using rotations and symmetries and is given on Fig. 1.

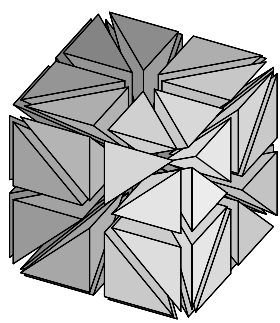

Fig. 1. The octaedral group $O_{h}$ associated to the decomposition of a cube into 48 tetraedra, each being a transformation of the fundamental domain $0 \leq a \leq b \leq c$ by an element of the group $O_{h}$

Using the octaedral group, we could study only the fundamental domain which is the subset $F$ of $\mathbb{Z}^{3}$ composed of the integer points $(a, b, c)$ such that $0 \leq a \leq$ $b \leq c$. To generate all possible cases, we simply study the action of $O_{h}$ on a triple of signed symbols $( \pm a, \pm b, \pm c)$. Each of the eight elements of the subgroup $\left(\frac{\mathbb{Z}}{2 \mathbb{Z}}\right)^{3}$ of $O_{h}$ modify the signs of the symbols and the other six, coming from $S_{3}$, permute them. Consequently, $O_{h}$ can be identified to the group of $3 \times 3$ matrices where 
each row and column contains only one value being either +1 or -1 . We can effectively find 48 of such matrices.

Let $n=\left(n_{1}, n_{2}, n_{3}\right) \in \mathbb{Z}^{3}$ be a vector and let us denote by $D_{0} m_{n}$ the domain of $n$. The element $g_{n}$ of $O_{h}$ which maps $D_{o m}$ onto $F$ can be constructed as follows. We sort the matrix whose columns are the $n_{i}$ such that the order of the first row is increasing. The resulting matrix is the element $M_{n}$. Let us now denote by perm $_{n}: \mathbb{Z}^{3} \rightarrow \mathbb{Z}^{3}$ and by $\operatorname{sgn}_{n}: \mathbb{Z}^{3} \rightarrow \mathbb{Z}^{3}$ the applications

$$
\operatorname{perm}_{n}:\left(\begin{array}{c}
x \\
y \\
z
\end{array}\right) \mapsto M_{n}\left(\begin{array}{l}
x \\
y \\
z
\end{array}\right) \quad \operatorname{sgn}_{n}:\left(\begin{array}{l}
x \\
y \\
z
\end{array}\right) \mapsto\left(\begin{array}{c}
\operatorname{sign}\left(n_{1}\right) x \\
\operatorname{sign}\left(n_{2}\right) y \\
\operatorname{sign}\left(n_{3}\right) z
\end{array}\right)
$$

where $\operatorname{sign}(w)$ is the sign of $w$. We have $g_{n}=\operatorname{sgn}_{n} \circ \operatorname{perm}_{n}$.

\section{3D Digital Lines}

We present in this section our construction of 3D digital lines using lattices of $\mathbb{R}^{n}$ and arithmetics. Recall that if $v_{1}, v_{2}, \ldots, v_{p}$ is a collection of $p$ vectors of $\mathbb{R}^{n}$, then the lattice generated by the collection is the set of all integral combinations

$$
\sum a_{i} v_{i}, \quad \forall i=1,2 \ldots, p, \quad a_{i} \in \mathbb{Z}
$$

Our approach is based on the study of the repartition of integer points of $\mathbb{Z}^{3}$ in the neighborhood of the integral direction given by the vector $(a, b, c)$. We will define $3 \mathrm{D}$ digital lines based on the notion of $1 \mathrm{D}$ dotted lines as it is the case for the two dimensional lines [13. Using the octaedral group $O_{h}$, we suppose that $(a, b, c)$ belongs to the fundamental domain $F$. Moreover, $a, b$ and $c$ are supposed to be relatively prime.

Let us denote by $\mathcal{E}$ the set of all Euclidean lines whose direction vector is $v$ and which contain integer points. We will call $1 D$ dotted lines with direction $v=(a, b, c)$, the intersection of the Euclidean lines - with direction $v$ - with $\mathbb{Z}^{3}$. The plane $(P)$ given by $a x+b y+c z=0$ is a subgroup of $\mathbb{R}^{3}$. The orthogonal projection of $\mathbb{Z}^{3}$ onto $(P)$ is the intersection of $\mathcal{E}$ and $(P)$ and is a lattice of $(P)$ denoted by $\mathcal{E}_{\mathcal{P}}$. This lattice is clearly a rational lattice (see Fig. 4).

It is easy to verify that the intersection of the planes $-c x+a z=0$ and $-c y+b z=0$ is the line directed by $v$ and passing through the origin. The intersections of the planes $-c x+a z=k$ and $-c y+b z=l$ where $k, l \in \mathbb{Z}$ also give a family of lines of direction $v$. We denote by $\mathcal{D}$ this family. It is clear that $\mathcal{E} \subset \mathcal{D}$, but the converse is false as the following system shows it,

$$
\left\{\begin{array}{l}
13 x-3 z=2 \\
13 y-5 z=3
\end{array}\right.
$$

corresponds to the line whose direction is $(3,5,13)$ but this line does not intersect $\mathbb{Z}^{3}$. Indeed, the solution of (1) are given by $(2+3 \mu, 0,8+13 \mu), \mu \in \mathbb{Z}$, whereas the solutions of $(2)$ are $(0,6+5 \nu, 15+13 \nu), \nu \in \mathbb{Z}$. To have an integer solution 
of the system, one must have: $\exists \mu, \nu \in \mathbb{Z}, 8+13 \mu=15+13 \nu$; this is clearly impossible.

Let us denote by $\mathcal{D}_{\mathcal{P}}$ the lattice given by the intersection of $\mathcal{D}$ and the plane $(P)$. It is clear that $\mathcal{E}_{P} \subset \mathcal{D}_{P}$. The lattice $\mathcal{E}_{P}$ belonging to $(P)$ is the projection of all $1 D$ dotted lines, but its use is not very easy. However, we can see it as a sublattice of $\mathcal{D}_{P}$. Since $\mathcal{D}_{P}$ is a Cartesian lattice, it is much easier to work with. These lattices were introduced in 2 to propose a new definition of $3 D$ digital lines. Beside this definition, we can refer to definition 4.2.3 of Voss [1] of $n D$ digital lines. In the sequel, we explain how to obtain Voss definition using two specific lattices whose construction is similar to the one of $\mathcal{E}_{P}$ and $\mathcal{D}_{P}$ of [2].

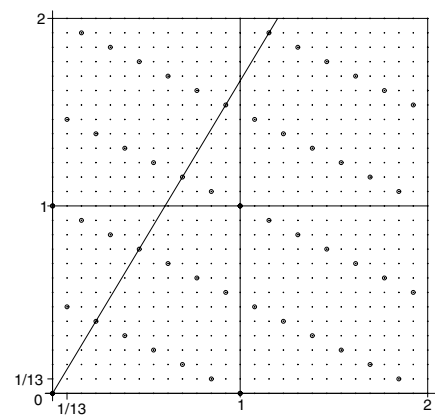

Fig. 2. The lattices $\mathcal{D}_{x y}, \mathcal{E}_{x y}$, the line $l$ with direction $(a, b, c)=(3,5,13)$

\subsection{The Lattices $\mathcal{E}_{x y}$ and $\mathcal{D}_{x y}$}

Let us denote by $\mathcal{E}_{x y}$ and $\mathcal{D}_{x y}$ the lattices which are respectively the intersections of the collection of lines $\mathcal{D}$ and $\mathcal{E}$ with the $x O y$ plane (see Fig. 2).

Proposition 1. The lattice $\mathcal{D}_{x y}$ is the set of integer points of the $x O y$ plane, given by $\left(\frac{k}{c}, \frac{l}{c}\right)$ where $k$ and $l$ are arbitrary integers.

Let $\mathcal{L}$ be the line with direction $v$ containing the origin and let $l$ be its projection onto the plane $z=0$. We then have the following.

Proposition 2. The lattice $\mathcal{E}_{x y}$ is the set of rationnal points $\left(x-\frac{a z}{c}, y-\frac{b z}{c}\right)$ of the plane $z=0$ where $x, y, z$ are arbitrary integers.

To efficiently manipulate 3D digital lines, we must clearly understand the lattice $\mathcal{E}_{x y}$. To do this, we now give a modular generation of this last lattice.

When $z$ varies in $\mathbb{Z}$, the points $\left(x+\frac{z a}{c}, y+\frac{z b}{c}\right)$ are located into unit squares given by $\left[k, k+1\left[\times\left[l, l+1\left[\subset \mathbb{R}^{2}\right.\right.\right.\right.$ where $k$ and $l$ are well chosen. We can consider the reduction of this series modulo $(1,1)$, that is $\left(\frac{k a \bmod c}{c}, \frac{k b \bmod c}{c}\right)$. We simplify the notation by denoting by $\left\{\frac{u}{v}\right\}$ the value of $u \bmod v$, such that the previous couple is $\frac{1}{c}(\{k a / c\},\{k b / c\})$. 
The $c$ points $\frac{1}{c}(\{k a / c\},\{k b / c\}), k=0,1,2, \ldots, c-1$ of the lattice $\mathcal{E}_{x y}$ all belong to the unit square $\left[0,1\left[\times\left[0,1\left[\right.\right.\right.\right.$. We denote by $\Pi_{a b c}$ this set. The whole lattice $\mathcal{E}_{x y}$ is obtained by periodic translations of $\Pi_{a b c}$. The only consideration of the lattices $\mathcal{D}_{x y}$ and $\mathcal{E}_{x y}$ will lead to the notion of $3 \mathrm{D}$ digital lines.

First, the points $\frac{1}{c}(z a, z b)$ of $\mathcal{E}_{x y}$, belonging to $l$, are the projections of the intersections of $\mathcal{L}$ with the planes $z=$ cste. But $l$ also intersects the unit squares of $\mathbb{R}^{2}$ given by $[x, x+1[\times[y, y+1[$. These squares are the projection over the plane $z=0$ of the voxels $\left[x, x+1\left[\times\left[y, y+1\left[\times\left[z, z+1\left[\right.\right.\right.\right.\right.\right.$ of $\mathbb{R}^{3}$. Consequently, the study of the intersections of $\mathcal{L}$ with the unit cube of $\mathbb{R}^{3}$ is equivalent to the study of the intersections of $l$ with the squares $[x, x+1[\times[y, y+1[$ and the study of the lattices $\mathcal{D}_{x y}$ and $\mathcal{E}_{x y}$.

If we consider the parallel lines to $\mathcal{L}$ containing a point of $\Pi_{a b c}$, thus the $1 D$ dotted lines with direction $v$, we can verify that they intersect the same voxels or neighboor voxels than $\mathcal{L}$. Hence, the set of intersected voxels is a 26 -connected structure we can call a $3 \mathrm{D}$ digital lines.

Among the points of $\mathcal{E}_{x y}$ belonging to $l$, which are generally rational points, the one given by $k(a, b)=\frac{k c}{c}(a, b)$ with $k \in \mathbb{Z}$ are integer points. These are the projections of the integer points $k(a, b, c), k \in \mathbb{Z}$ belonging to the line $\mathcal{L}$.

Theorem 1. The projection of $k(a, b, c), k \in \mathbb{Z}$ of the line $\mathcal{L}$ is the series of integer points of the plane $z=0$ given by $k(a, b), k \in \mathbb{Z}$. If $\frac{1}{c}(m, n)$ is a point different from $(0,0)$ in $\Pi_{a b c}$, either $i=-m a^{-1} \bmod c$ or $i=-n b^{-1} \bmod c$ where $a^{-1}$ (resp. $\left.b^{-1}\right)$ is the inverse of a (resp. b) in the group $\frac{\mathbb{Z}}{c \mathbb{Z}}$, then $\left(\frac{1}{c}(m+\right.$ $\left.i a), \frac{1}{c}(n+i b)\right)$ is an integer point and is the projection of the point $\left(1+\left[\frac{i a}{c}\right], 1+\right.$ $\left.\left[\frac{i b}{c}\right], i\right)$ of the dotted line parallel to $\mathcal{L}$ and containing the point $(m, n)$.

Proof. The first relation on $\mathcal{L}$ has already been given. For the second on an arbitray dotted lines, as the three integers $a, b, c$ are relatively prime $((a, b, c)=1$ where $(a, b, c)$ is the gcd of the three numbers), then $a$ or $b$ is invertible $\bmod c$ (as $(m, n) \in \mathcal{E}_{x y}, m a^{-1}=n b^{-1} \bmod c$ if both are invertible). Consequently, $m+i a$ and $n+i b$ are multiples of $c$. Using the Euclidean division between $m+i a$ and $n+i b$, we obtain the last relation of the theorem.

We now describe the construction of the $3 \mathrm{D}$ digital lines $\Delta_{a b c}$ directed by $v$ and containing the origin. This line is an union of $c 1 \mathrm{D}$ dotted lines and $\mathcal{L}$ is one of them. We then apply theorem 1 to add one by one 1D dotted lines to $\Delta_{a b c}$.

We consider the point $\frac{1}{c}(m, n)=\frac{1}{c}((c-1) a,(c-1) b)$ of $\Pi_{a b c}$ and the line $\delta$ directed by $(a, b)$ and containing $\frac{1}{c}(m, n)$. The sum $\left.\frac{1}{c}(m, n)+\frac{1}{c}(a, b)\right)$ is equal to $(a, b)$, hence the point following $\frac{1}{c}(m, n)$ on $\delta$ is an integer point which is the projection of the point $(1,1,1)$ (here $i=1)$. We obtain thus that the $3 \mathrm{D}$ line directed by $v$ and containing the point $(1,1,1)$ is parallel to $\mathcal{L}$. It is also a $1 \mathrm{D}$ dotted line whose integer points are $(1,1,1)+k(a, b, c), k \in \mathbb{Z}$. We add it to $\Delta_{a b c}$.

By adding the vector $\frac{1}{c}(2 a, 2 b)$ to the point $\frac{1}{c}(m, n)=\frac{1}{c}((c-2) a,(c-2) b)$ of $\Pi_{a b c}$, we also obtain an integer point $(x, y)$ of $\mathcal{E}_{x y}$ (equal to $(1,1)$ or $(1,2)$ depending on the relative values of $a, b, c)$. This point $(x, y)$ is the projection of the point $(x, y, 2)$ of $\mathbb{Z}^{3}$ defining the $1 \mathrm{D}$ dotted line made by the points $(x, y, 2)+$ $k(a, b, c), k \in \mathbb{Z}$. We also add it to $\Delta_{a b c}$. 
We pursue this construction by adding all the dotted lines defined by the points $\frac{1}{c}((c-i) a,(c-i) b)$ of $\Pi_{a b c}$. At the end, when $i=c$, we obtain an integer point of $\mathcal{L}$. Hence, $\Delta_{a b c}$ is periodic with period $(a, b, c)$. Its period is composed of the $c$ first voxels described previously. As $(c-i) a=-i a \bmod c$, it is easy to compute the $x$ and $y$ coordinates of the voxels.

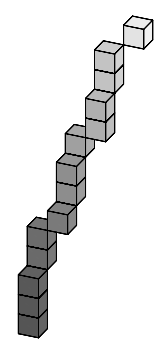

Fig. 3. The first 13 voxels of the first period followed by the first voxel of the second one. The direction vector is $(a, b, c)=(3,5,13)$.

We thus obtain Voss definition of a 3D digital lines (see Fig. 33).

Definition 1. The $3 D$ digital line with direction $(a, b, c)$ and initial conditions $m, n$ at the origin, where $0 \leq m<c$ and $0 \leq n<c$, is given by

$$
\left\{\begin{array}{l}
x=\left[\frac{a z+m}{c}\right] \\
y=\left[\frac{b z+n}{c}\right] \quad \text { with } \quad z \in \mathbb{Z} . \\
z=z
\end{array}\right.
$$

Contrarily to the $2 \mathrm{D}$ case, choosing $m=n=\frac{c}{2}$ does not produce the approximation with rounding of the Euclidean line. In fact, we will recall that this Bresenham-like $3 \mathrm{D}$ digital line is generated via the lattices $\mathcal{E}_{P}$ and $\mathcal{D}_{P}$.

From the previous study, it becomes easy to find an algorithm to draw the $3 \mathrm{D}$ digital lines. Indeed, if we translate the point $\frac{1}{c}(m, n) \in \Pi_{x y}$ by the vector $(a, b), 4$ cases happen

$$
\begin{cases}0 \leq m<c-a \text { and } 0 \leq n<c-b & \text { step }(0,0,1) \\ c-a \leq m \text { and } 0 \leq n<c-b & \text { step }(1,0,1) \\ 0 \leq m<c-a \text { and } c-b \leq n & \text { step }(0,1,1) \\ c-a \leq m \text { and } c-b \leq n & \text { step }(1,1,1)\end{cases}
$$

The $3 D$ digital lines, previously defined, with direction vector $n=(a, b, c)$ such that $0 \leq a<b<c$ and $a, b, c$ relatively prime, are given by the intersection of two particular digital planes.

Definition 2. A $3 D$ digital lines with direction vector $(a, b, c)$ such that $0 \leq a<$ $b<c$ and $a, b, c$ relatively prime is the set of solutions of the linear systems of inequalities given by 


$$
\left\{\begin{array}{l}
\gamma \leq c x-a z<\gamma+c \\
\gamma^{\prime} \leq c y-b z<\gamma^{\prime}+c
\end{array}\right.
$$

The lower bounds can be used to translate the digital line so that it can contain any given integer point of $\mathbb{Z}^{3}$. Their arithmetical thickness is the integer $c$. This number can be replaced by any couple $\epsilon, \epsilon^{\prime}$ of integers in orderto define $3 D$ digital lines with prescribed thickness.

One on the fundamental properties of $3 d$ digital lines is that they cover $\mathbb{Z}^{3}$. Moreover, the action of the octahedral group $O_{h}$ can be used to define $3 D$ digital lines with any direction vector.

\subsection{The Lattices $\mathcal{D}_{P}$ and $\mathcal{E}_{P}$}

Recall that for a direction vector $n=(a, b, c)$, the canonical lattices attached to the famillies $\mathcal{D}$ and $\mathcal{E}$ are the intersections of these famillies with the plane $(P): a x+b y+c z=0$. The lattices are respectively $\mathcal{D}_{P}$ and $\mathcal{E}_{P}$.

The lattice $\mathcal{E}_{P}$ is very interesting since it permits to measure Euclidean distances between the 1D dotted lines so to locate the integer points which are closest to an Euclidean lines with direction vector $n$. As it was the case for $\mathcal{D}$, $\mathcal{D}_{P}$ is a cartesian lattice which contains $\mathcal{E}_{P}$ and with which it is easier to work.

The coordinates of the points of $\mathcal{D}_{P}$ and $\mathcal{E}_{P}$ are more complex that for $\mathcal{D}_{x y}$ and $\mathcal{E}_{x y}$ but their dependances are algegraically similar. We refer to 2 for the computation. The coordinates of the points of $\mathcal{D}_{P}$ are given by:

$$
\left\{\begin{array}{rl}
x & =\frac{\left(b^{2}+c^{2}\right) u-a b v}{a^{2}+b^{2}+c^{2}} \\
y & =\frac{\left(a^{2}+c^{2}\right) v-a b u}{a^{2}+b^{2}+c^{2}} \\
z & =\frac{-c(a u+b v)}{a^{2}+b^{2}+c^{2}}
\end{array} \quad u, v \in \mathbb{Z}\right.
$$

Hence, $\mathcal{D}_{P}$ is generated by the vectors $\alpha=\frac{1}{a^{2}+b^{2}+c^{2}}\left(b^{2}+c^{2},-a b,-a c\right)$ and $\beta=\frac{1}{a^{2}+b^{2}+c^{2}}\left(-a b, a^{2}+c^{2},-b c\right)$.

The lattice $\mathcal{E}_{P}$ is generated by the reductions modulo $\alpha$ and $\beta$ of the vectors $\frac{k}{a^{2}+b^{2}+c^{2}}\left(a c, b c,-\left(a^{2}+b^{2}\right)\right), \quad k \in \mathbb{Z}$. Fig. 4 shows a partial view of a lattice $\mathcal{E}_{P}$ as well as several $1 \mathrm{D}$ dotted lines of $\mathcal{E}$.

Both $\mathcal{D}_{P}$ and $\mathcal{E}_{P}$ are planar lattice with rank 2. Then, using a convenient isometry we can map them onto $x O y$. After some tedious calculus, the isometric lattice of $\mathcal{D}_{P}$ is generated by the vectors $U=\frac{1}{\sqrt{\left(a^{2}+b^{2}\right)}}\left(1, \frac{-a b}{c \sqrt{\left(a^{2}+b^{2}+c^{2}\right)}}\right)$ and $V=\frac{a^{2}+c^{2}}{c \sqrt{\left.\left(a^{2}+b^{2}\right) \sqrt{(} a^{2}+b^{2}+c^{2}\right)}}(0,1)$. The image of the lattice $\mathcal{E}_{P}$ is the reduction modulo $U$ and $V$ of the vectors $k(a U, b V) \quad k \in \mathbb{Z}$. As it can be easily seen, this situation is the analoguous of the link between $\mathcal{D}_{x y}$ and $\mathcal{E}_{x y}$.

Given a point in $\mathcal{E}_{P}$, the closest points in $\mathcal{E}_{P}$ to the given points enables us to define the notion of closest $3 D$ digital lines (see Fig. 5). This corresponds to a Bresenham-like $3 D$ digital lines. To define it, one must sort the points in $\mathcal{E}_{P}$ around a given point in $\mathcal{E}_{P}$

Let $\pi: \mathbb{Z}^{3} \mapsto \mathcal{E}_{P}$ be the application which maps a $1 \mathrm{D}$ dotted line to its intersection with the plane $(P)$, let $\omega$ be a point of $\mathcal{E}_{P}$ and let $\Delta_{\omega, \rho}$ be the set 


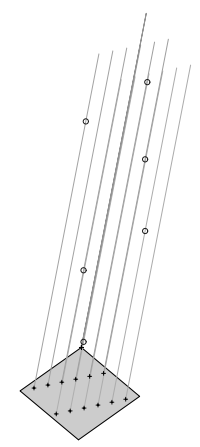

Fig. 4. A part of the lattice $\mathcal{E}_{P}$ where $(a, b, c)=(3,5,13)$ as well as some 1D dotted lines

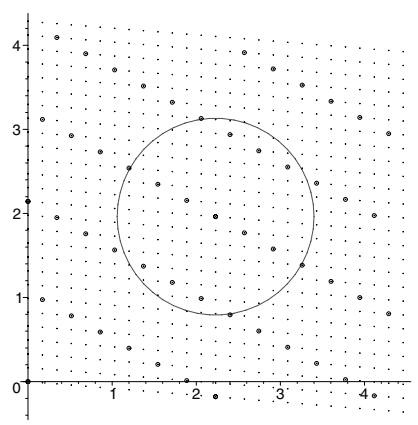

Fig. 5. The isometric images of $\mathcal{D}_{P}$ and $\mathcal{E}_{P}$, where $(a, b, c)=(3,5,13)$, in the plane $x O y$ and a circle of radius 1.17 containing some closest points of one element of $\mathcal{E}_{P}$

of points in $\mathcal{E}_{P}$ belonging to a disk (in plane $(P)$ ) with center $\omega$ and radius $\rho$, then we have the following,

Definition 3. The $3 D$ digital line with best integer approximation of order $\rho$ of the $1 D$ dotted line containing the point $\omega$ of $\mathcal{E}_{P}$ is the reciprocical image $\pi^{-1}\left(\Delta_{\omega, \rho}\right)$.

Obviously, these $3 D$ digital lines does not cover $\mathbb{Z}^{3}$, which could be a bad behaviour. Nevertheless, it guarantees that the digital lines is as closest as possible of the Euclidean corresponding line.

\subsection{D Segment Drawing}

If any segment $A B$ is given let us denote by $O V$ the vector $B-A$ and by $n=\left(n_{1}, n_{2}, n_{3}\right)$ the components of $O V$ divided by their greatest common divider, so that $n_{1}, n_{2}, n_{3}$ are relatively prime. Construction given in section 3 about the symmetry group $O_{h}$ can be used to give an operator $g_{n}$ mapping the domain of 
$n$ (or $O C$ ) to the fundamental domain $F$ of $O_{h}$. Let $n^{\prime}=g_{n} . n=(a, b, c)$ and $O C^{\prime}=g_{n} . O C=\left(u_{1}^{\prime}, u_{2}^{\prime}, u_{3}^{\prime}\right)$ in $F$.

Operator $g_{n}^{-1}$ followed by the translation of $O C$ to $A B$ leads to a procedure $g(x, y, z)$ which maps the $3 D$ discrete segment associated with $O C^{\prime}$ to the one associated to $A B,\left(g_{n}\right.$ being orthogonal $g_{n}^{-1}$ is equal to the transposed of $\left.g_{n}\right)$. Drawing the $3 D$ discrete segment associated to $A B$ is thus reduced to the following algorithm giving the discrete approximation of $O C^{\prime}$ directed by $n^{\prime}=(a, b, c)$.

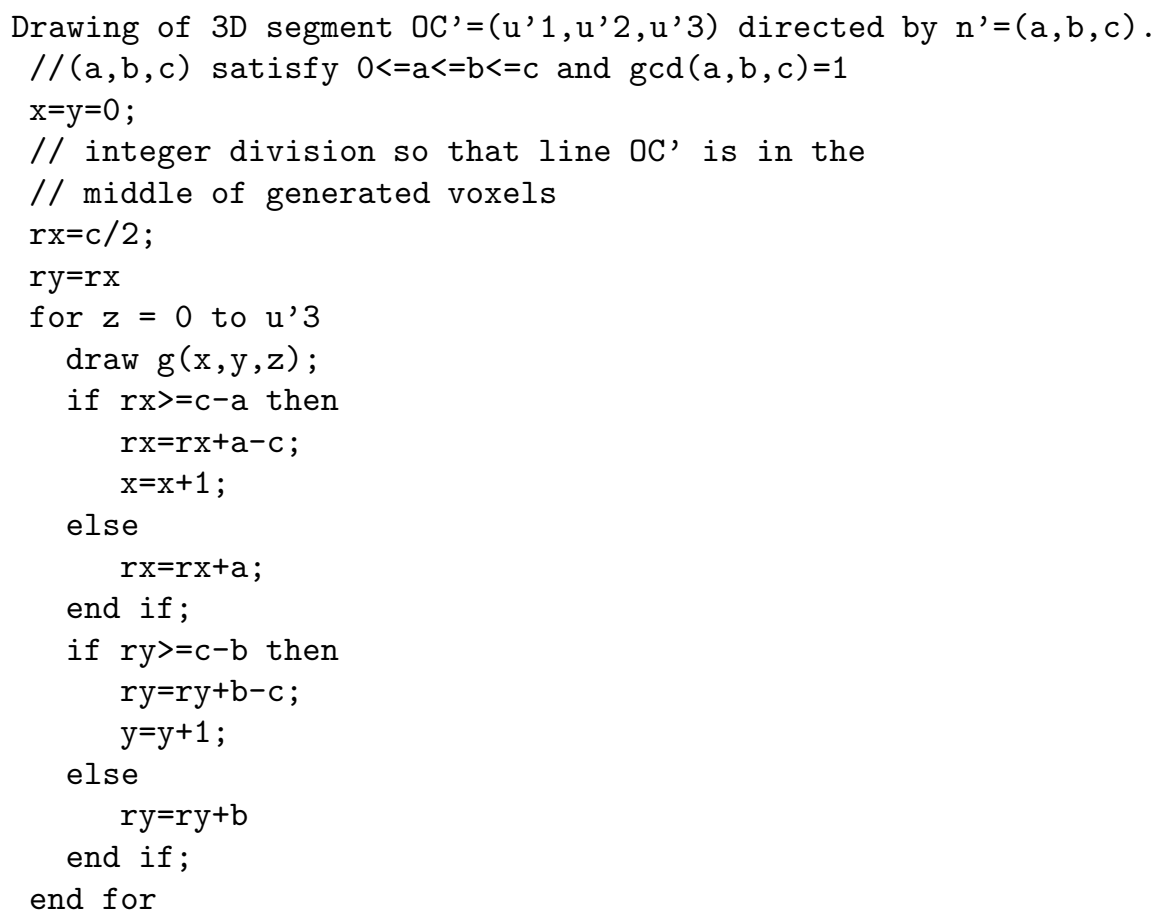

\section{$4 \mathrm{nD}$ Digital Lines}

Let $v=\left(a_{1}, a_{2}, \ldots, a_{n}\right) \in F$ an integer point in the fondamental domain of the hyperoctaedral group $\mathcal{B}_{n}$. This group of order $2^{n} . n$ ! can be identified with integer matrices of order $n$ where each row and column contains one and only one non-zero term equals to \pm 1 .

Let $P$ be the hyperplane whose equation is $a_{1} x_{1}+a_{2} x_{2}+\ldots a_{n} x_{n}=0$, and $E_{P}$ be the lattice obtained by projection of $\mathbb{Z}^{n}$ on $P$ along direction $v$. We denote by $\left\{u_{i}\right\} \quad 1 \leq i \leq n$ the canonical basis of $\mathbb{Z}^{n}$ and $X_{i}$ the projection of $u_{i}$ onto $P$ along $v$. Vectors $X_{i}$ belong to $E_{P}$ and from equation of hyperplane $P$ we have:

$$
X_{n}=a_{1}\left(\frac{X_{1}}{a_{n}}\right)+a_{2}\left(\frac{X_{2}}{a_{n}}\right)+\cdots+a_{n-1}\left(\frac{X_{n-1}}{a_{n}}\right)
$$

We consider the lattice $D_{P}$ generated in $P$ by the $n-1$ vectors $\frac{X_{1}}{a_{n}}, \frac{X_{2}}{a_{n}}, \ldots$, $\frac{X_{n-1}}{a_{n}}$; of course $E_{P}$ is a sublattice of $D_{P}$. Moreover, $E_{P}$ is $n-1$ periodic, one 
period being given by $E_{P} \cap \delta, \delta$ denoting the fondamental domain of $D_{P}$. Any point of $E_{P}$ is obtained by reduction modulo $\delta$ of integer multiples of $X_{n}$.

Let $\sigma^{2}=a_{1}^{2}+a_{2}^{2}+\cdots+a_{n}^{2}$ and $\widehat{\sigma_{i}^{2}}=a_{1}^{2}+a_{2}^{2}+\cdots+\widehat{\left(a_{i}^{2}\right)}+\cdots+a_{n}^{2}$ where, in the sum $\widehat{u}$ means omission (so that $\widehat{\sigma_{i}^{2}}=\sigma^{2}-a_{i}^{2}$ ), then components $x_{i j}$ of $X_{i}=\left(x_{i j}\right)$ can be computed and we have $x_{i j}=\frac{-a_{i} a_{j}}{\sigma^{2}}$ if $i \neq j$ and $x_{i i}=\frac{\widehat{\sigma_{i}^{2}}}{\sigma^{2}}$ for $i=j$.

From these expressions of vectors $X_{i}$ the determinant of the Gram matrix of $D_{P}$ can be evaluated giving $\operatorname{det}\left(\left(X_{i} . X_{j}\right)\right)=a_{n}$ showing that domain $\delta$ contains $a_{n}$ elements of $E_{P}$.

The hypothesis $v \in F$ leads to a very natural observation which will be helpful to define $n D$ digital lines. Computation of the norm of $X_{i}-X_{j}$ gives $\left\|X_{i}-X_{j}\right\|^{2}=\frac{\widehat{\sigma_{i}^{2}}+\widehat{\sigma_{j}^{2}}+\left(a_{i}+a_{j}\right)^{2}}{\sigma^{2}}$ from which inequalities $\left\|X_{i}\right\| \leq\left\|X_{i}-X_{j}\right\|$ and $\left\|X_{j}\right\| \leq\left\|X_{i}-X_{j}\right\|$ can be deduced showing that the set $\left\{X_{1}, X_{2}, \ldots X_{n-1}\right\}$ is almost orthogonal in $D_{P}$.

Let $\Pi_{i}$ be the hyperplane generated by $u_{1}, \ldots, \widehat{u_{i}}, \ldots, u_{n-1}, v$ where, again, - means omission. Intersection $\Pi_{i} \cap P$ is the subspace of $P$ generated by $X_{1}, \ldots, \widehat{X}_{i}, \ldots, X_{n-1}$ so that these hyperplanes too are almost orthogonal. Definition of digital hyperplanes in $\mathbb{Z}^{n}$ being obvious we can define $n D$ digital line through 0 and directed by $v$ as the intersection of digital hyperplanes associated to $\Pi_{i}^{\prime} s$.

Definition 4. Digital line through 0 directed by $v=\left(a_{1}, a_{2}, \ldots, a_{n}\right)$ where $0 \leq$ $a_{1} \leq a_{2} \leq \cdots \leq a_{n}$ is the set of integer points solution of the $n-1$ diophantine inequations

$$
\gamma_{i} \leq a_{1} x_{1}+\cdots+\widehat{\left(a_{i} x_{i}\right)}+\ldots a_{n-1} x_{n-1}+a_{n} x_{n}<\gamma_{i}+\epsilon_{i} \quad 1 \leq i \leq n-1
$$

$\widehat{-}$ meaning omission.

Vector $\left(\gamma_{i}\right)$ is the lower bound and vector $\left(\epsilon_{i}\right)$ the arithmetical thickness.

Algorithms can be given to draw digital $n D$ lines defined in this way. They use a vector of errors $\rho=\left(r_{1}, r_{2} \ldots r_{n-1}\right.$ and the simplest one draws $2^{n}-1$ connected lines when $\epsilon_{i}=a_{n} \forall i=1,2, \ldots n-1$; again we suppose $v \in F$, the general case being solved with the help of operators of the Hyperoctaedral group $\mathcal{H} \backslash$ in a similar way as what has been donne in $3 D$.

Suppose $M=\left(m_{1}, m_{2}, \ldots, m_{n}\right)$ is a point in $\mathbb{Z}^{n}$ and $v=\left(a_{1}, a_{2}, \ldots, a_{n}\right) \in F$ and $\operatorname{gcd}\left(a_{i}\right)=1$, then to obtain the first nbPoints of the $n D$ and $2^{n}-1$-connected digital line through $M$ and directed by $v$ we have the following algorithm.

$\mathrm{nD}$ digital line drawing

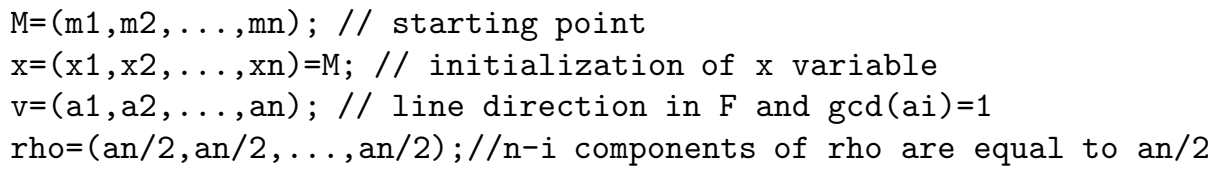




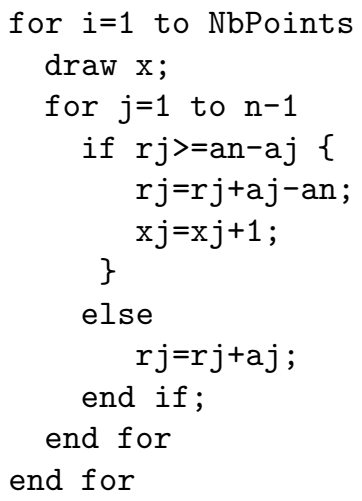

Initializing error vector $\rho$ with half of thickness, that is setting $\frac{a_{n}}{2}$ for all $\rho$ 's component we are assured that integer points generated are well distributed around the euclidean line going through $M$ and directed by $v$.

Following array shows an application of this algorithm for the drawing of the first 13 points of the $4 D$ digital line going through origin and directed by vector $v=(3,5,7,13)$. First 3 lines show evolution of $\rho=\left(r_{1}, r_{2}, r_{3}\right)$ error vector and the last ones are coordinates of approximating points. One period of this line is thus obtained; following ones are obtained by translating this one by integer multiples of vector $v$.

$\begin{array}{cccccccccccccccc}r_{1} & 6 & 9 & 12 & 2 & 5 & 8 & 11 & 1 & 4 & 7 & 10 & 0 & 3 & 6 \\ r_{2} & 6 & 11 & 3 & 8 & 0 & 5 & 10 & 2 & 7 & 12 & 4 & 9 & 1 & 6 \\ r_{3} & 6 & 0 & 7 & 1 & 8 & 2 & 9 & 3 & 10 & 4 & 11 & 5 & 12 & 6 \\ & & & & & & & & & & & & & \\ x_{1} & 0 & 0 & 0 & 1 & 1 & 1 & 1 & 2 & 2 & 2 & 2 & 3 & 3 \\ x_{2} & 0 & 0 & 1 & 1 & 2 & 2 & 2 & 3 & 3 & 3 & 4 & 4 & 5 \\ x_{3} & 0 & 1 & 1 & 2 & 2 & 3 & 3 & 4 & 4 & 5 & 5 & 6 & 6 \\ x_{4} & 0 & 1 & 2 & 3 & 4 & 5 & 6 & 7 & 8 & 9 & 10 & 11 & 12\end{array}$

\section{Conclusion}

We have presented in this paper a unified view of the definitions of Voss [1] and Figueiredo and Reveillès [2]. This permits us, for instance, to give a short drawing algorithm in 3D. Moreover, the presentation is extended to an arbitrary dimension via the use of lattice theory and specific projections. We also give a 13-lines long drawing algorithm for $\mathrm{nD}$ digital lines. It should be very interesting to study the link between this approach and multi-dimensonal continued fraction given by Arnold [14] and this is a future work.

\section{References}

1. Voss, K.: Discrete Images, Objects and Functions in $\mathbb{Z}^{n}$. Springer-Verlag (1993)

2. Figueiredo, O., Reveillès, J.P.: New results about 3D digital lines. In Melter, R.A., Wu, A.Y., Latecki, L., eds.: Vision Geometry V. Volume 2826. (1996) 98-108 
3. Klette, R., Rosenfeld, A.: Digital Geometry. Morgan-Kaufmann (2004)

4. Kim, C.: Three-dimensional digital line segments. IEEE Trans. Pattern Analysis and Machine Intelligence 5 (1983) 231-234

5. Debled-Rennesson, I.: Etude et reconnaissance des droites et plans discrets. PhD thesis, Université Louis Pasteur - Strasbourg (1995)

6. Courjolly, D., Debled-Rennesson, I., Teytaud, O.: Segmentation and length estimation of 3D discrete curves. In Bertrand, G., et al, eds.: Digital and Image Geometry. Volume 2243 of LNCS., Springer-Verlag (2001) 299-317

7. Reveillès, J.P.: Géométrie discrète, calcul en nombres entiers et algorithmique. Thèse d'etat, Université Louis Pasteur, Strasbourg (1991)

8. Figueiredo, O., Reveillès, J.P.: A contribution to 3D digital lines. In: Proc. Discrete Geometry for Computer Imagery, Université d'Auvergne - LLAIC (1995) 187-198

9. Ibanez, L., Hamitouche, C., Roux, C.: A Vectorial Algorithm for Tracing Discrete Straight Lines in N-Dimensional Generalized Grids. IEEE Trans. Vis. Comput. Graph. 7(2) (2001) 97-108

10. Klette, R.: The m-dimensional grid point space. Computer Vision, Graphics, and Image Processing 30 (1985) 1-12

11. Stojmenovic, I., Tosic, R.: Digitization Schemes and the Recognition of Digital Straight Lines, Hyperplanes, and Flats in Arbitrary Dimensions. Contemporary Mathematics 119 (1991) 197-212

12. Reveillès, J.P.: The Geometry of the Intersection of Voxel Spaces. In Fourey, S., Herman, G., Kong, T.Y., eds.: IWCIA 2001. Volume 46 of Electronic Notes in Theoretical Computer Science., Elsevier (2001) 1-24

13. Debled, I., Reveillès, J.: A linear algorithm for segmentation of digital curves. In: $3^{\text {rd }}$ IWPIA. (1994)

14. Arnold, V.: Higher dimensional Continued Fractions. Regular and Chaotics Dynamics 33 (1998) 10-17 